\title{
Towards the Thinking Microscope
}

\author{
Oleg S. Ovchinnikov, ${ }^{*} * *$ S. Jesse, ** S.V. Kalinin, H. J. Chang, ${ }^{* *}$ \\ S.J. Pennycook, ** A.Y. Borisevich** \\ *University of Tennessee, Knoxville \\ **Oak Ridge National Laboratory, PO Box 2008, Oak Ridge, TN 37831
}

The capability of electron microscopy and spectroscopy to examine structure and chemical composition from mesoscopic to atomic scales makes it the ultimate characterization tool for condensed matter physics and materials science. In particular, electron energy loss spectroscopy (EELS) in the scanning transmission electron microscope (STEM) allows for spatially resolved studies of composition, electronic and magnetic structure (in the core-loss region), as well as elementary excitations such as plasmons (in the low-loss region). Ideally, structural information obtained via STEM imaging (bright-field and dark field) can be synergistically combined with EELS spectroscopic data, providing a direct correlation between structure and electronic functionality. With aberration-corrected electron optics and improved EELS hardware, such correlations can be examined in unprecedented detail.

However, progress in this field is strongly hindered by the critical bottleneck of analysis and interpretation of the 3- and higher dimensional datasets (spectrum images) with millions of data points, their registration with the 2D structure images, and interpretation in terms of desired materials functionality. The current method of analyzing such data relies on a large amount of human input on every step of the process. This not only makes the process of analyzing the data extremely time consuming but also tends to introduce error as well as user bias into the data analysis. By automating the process of data analysis it will be possible to increase efficiency and reduce artifacts.

In this presentation, I will describe the automated for STEM and EELS data classification and analysis based on the combination of grid search and data mining algorithms. As a first step, I have developed an approach for automatic indexing the atomic lattices. The local maxima are identified using erosion-expansion algorithm, and classified in a operator-defined number of classes (e.g. corresponding to the $\mathrm{A}$ and $\mathrm{B}$ cationic sublattices in perovskites). The reliably-determined lattice sites are used to determine the most likely lattice vectors, and then adaptive algorithm is used to find the remaining atoms. The example of this algorithm is shown in Figure 1. Thus indexed lattice is analyzed using position refinement using appropriate shape function (e.g. pseudoVoigt) to extract the atomic spacings, from which local strains (e.g. variation in lattice parameters), polarization (relative displacements of $\mathrm{A}$ and B sublattice), and octahedral tilts (from bright field dark field image pair) can be established. The atomic column shape is analyzed using shape PCA analysis to provide information on the octahedral distortions [1]. Currently, we are working on developing the algorithm to match the 2D image data to the 3D EELS data to enable direct recognition of atomic structures and fine details of electronic properties on atomic level, as exemplified by Figure 2 [2,3].

\section{References}

[1] arXiv:1002.2989v1 [cond-mat.mtrl-sci]

[2] A.Y. Borisevich et al., these proceedings

[3] This research is sponsored by the Division of Materials Sciences and Engineering, Office of BES of the U.S. DOE, 


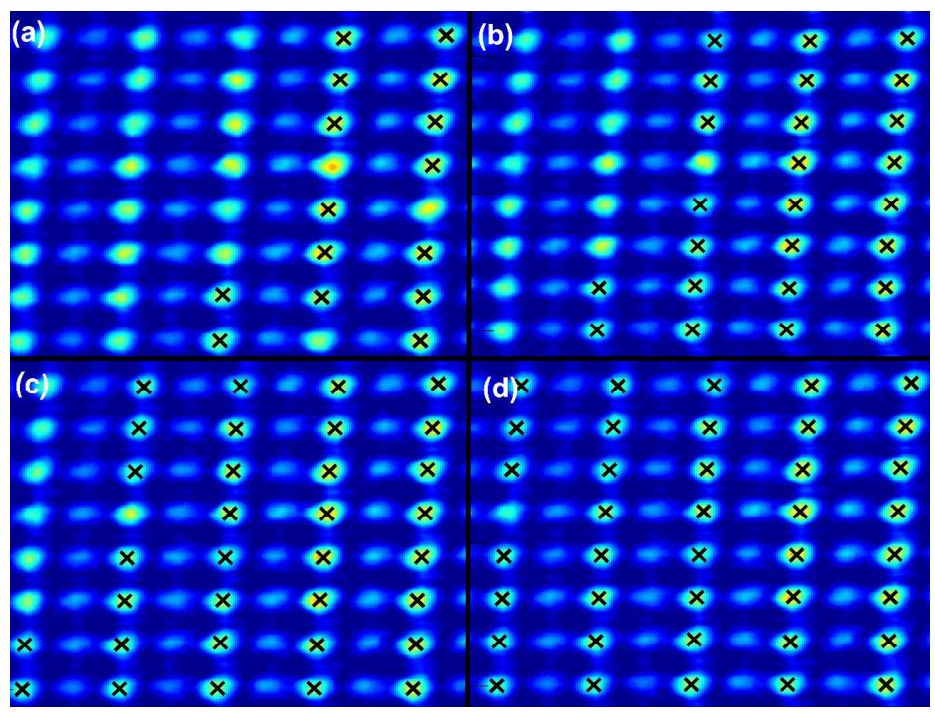

FIG. 1. Image of SrRuO3 lattice that has been de-noised and has had background removes through the use of image recognition techniques. (a) Initial centers of atoms found using image recognition. (b) Centers of atoms after one iteration of adding more centers based on know centers. (c) Centers of atoms after two iteration of adding more centers based on know centers. (d) Centers of atoms after three iteration of adding more centers based on know centers.
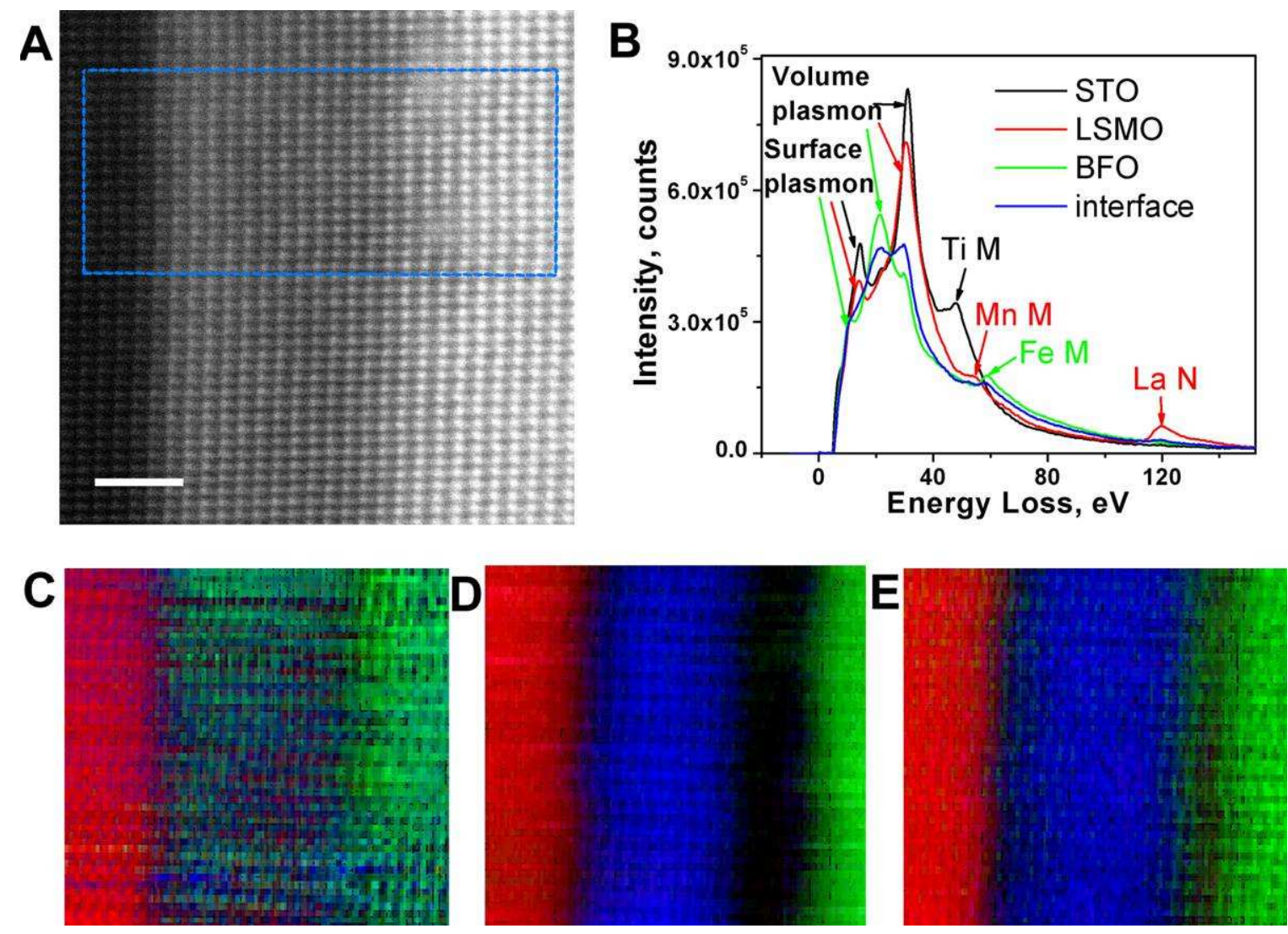

FIG 2. Low loss EELS imaging of the $\mathrm{SrTiO}_{3} /(\mathrm{La}, \mathrm{Sr}) \mathrm{MnO} 3 / \mathrm{BiFeO}_{3}$ interface. (a) imaging area; (b) representative spectra (after Fourier-log deconvolution) of the three components showing distinctive signatures and features; (c-e) resulting recognition maps for (c) zero loss region (-5 to $5 \mathrm{eV}$ ), (d) plasmon excitation region (5 to $30 \mathrm{eV}$ ), and (e) low-lying core edges ( 35 to $150 \mathrm{eV}$ ). Note that a region of BFO approximately $2 \mathrm{~nm}$ wide is not recognized as any of the components in map (d) (reproduced from[2]). 